\title{
Anatomical Versus Nonanatomical Resection of Colorectal Liver Metastases: Is There a Difference in Surgical and Oncological Outcome?
}

\author{
Zarina S. Lalmahomed $\cdot$ Ninos Ayez \\ Anne E. M. van der Pool · Joanne Verheij • \\ Jan N. M. IJzermans • Cornelis Verhoef
}

Published online: 16 December 2010

(C) The Author(s) 2010. This article is published with open access at Springerlink.com

\begin{abstract}
Background The increased use of neoadjuvant chemotherapy and minimally invasive therapies for recurrence in patients with colorectal liver metastases (CLM) makes a surgical strategy to save as much liver volume as possible pivotal. In this study, we determined the difference in morbidity and mortality and the patterns of recurrence and survival in patients with CLM treated with anatomical (AR) and nonanatomical liver resection (NAR).

Methods From January 2000 to June 2008, patients with CLM who underwent a resection were included and divided into two groups: patients who underwent AR, and patients who underwent NAR. Patients who underwent simultaneous radiofrequency ablation in addition to surgery and patients with extrahepatic metastasis were excluded. Patient, tumor, and treatment data, as well as disease-free and overall survival (OS) were compared.

Results Eighty-eight patients (44\%) received AR and 113 patients $(56 \%)$ underwent NAR. NAR were performed for significant smaller metastases ( 3 vs. $4 \mathrm{~cm}, P<0.001$ ). The Clinical Risk Score did not differ between the groups. After NAR, patients received significantly less blood transfusions $(20 \%$ vs. $36 \%, P=0.012)$, and the hospital stay
\end{abstract}

Z. S. Lalmahomed · J. N. M. IJzermans

Division of Transplantation and Hepatobiliary Surgery, Erasmus

University MC, Rotterdam, The Netherlands

N. Ayez · A. E. M. van der Pool · C. Verhoef $(\bowtie)$

Division of Surgical Oncology, Daniel den Hoed Cancer Center, Erasmus University MC, PO Box 5201, 3008 AE Rotterdam,

The Netherlands

e-mail: c.verhoef@erasmusmc.nl

J. Verheij

Division of Pathology, Erasmus University MC, Rotterdam,

The Netherlands was significantly shorter ( 7 vs. 8 days, $P<0.001)$. There were no significant differences in complications, positive resection margins, or recurrence. For the total study group, estimated 5-year disease-free and OS was 31 and 44\%, respectively, with no difference between the groups.

Conclusions Our study resulted in no significant difference in morbidity, mortality, recurrence rate, or survival according to resection type. NAR can be used as a save procedure to preserve liver parenchyma.

\section{Introduction}

Colorectal cancer is the most common gastrointestinal malignancy worldwide, affecting nearly one million people each year [1]. Half of these patients have or will develop hepatic metastases at some point during their life. Liver resection is considered to be the best treatment for colorectal liver metastases (CLM) with 5-year survival rates up to $60 \%$ in highly selected patients [2]. Until recently, only $10-20 \%$ of patients were considered suitable for attempted curative resection $[3,4]$. Due to improvements in surgical techniques, the acceptance of resection margins $<1 \mathrm{~cm}$, the introduction of more effective systemic chemotherapeutics, the use of portal vein embolization (VPE), the addition of radiofrequency ablation (RFA), and stereotactic body radiation (STBR) to surgery, more patients are eligible for liver surgery [5-13]. Moreover, the indications for liver resection have expanded during the past decade and there are only few limitations left, which include unresectable extrahepatic disease and insufficient future remnant liver. The question has shifted from "what can be resected" to "what will be left."

During this period, a change in surgical approach can be observed by an increase of nonanatomical resections [14]. A 
nonanatomical resection maximizes the amount of residual liver parenchyma, which is important, in particular for patients who received neoadjuvant chemotherapy. Although chemotherapy increases resectability, it is associated with hepatic changes, which might increase the risk of progressive hepatic failure and death after resection $[15,16]$. Moreover, in case of intrahepatic recurrences after partial liver resection in patients with CLM, a sufficient liver residual can offer the opportunity for local treatment [17].

Although anatomical hepatic resection has been reported to improve patient survival in hepatocellular carcinoma (HCC) [18-20], the literature about CLM is conflicting.

The purpose of this study was to investigate the influence of a nonanatomical liver resection (NAR) compared with an anatomical resection (AR) on morbidity, mortality, margin positivity, disease-free, and OS.

\section{Methods}

All patients who underwent partial hepatic resection for CLM at the Erasmus Medical Center from January 2000 to June 2008 were evaluated for inclusion in this study. Patients who underwent simultaneous AR and NAR or received additional RFA in addition to surgery as well as patients with extrahepatic metastasis were excluded.

Patients were divided into two groups: patients who underwent an AR, and patients who underwent a NAR. An AR was defined as resection of two or more hepatic segments as described by Couinaud [21]. This includes bisegmentectomy, (extended) right hemihepatectomy, (extended) left hemihepatectomy, or a combination of these [22]. NAR was defined as resection of the CLM, including a rim of microscopically normal tissue. The choice of resection type was made in a multidisciplinary hepatobiliary working group, based on tumor number, location, and patient status.

Information collected included demographic details, primary tumor stage (TNM-classification), maximum size, number and distribution of liver metastases on CT, plasma carcinoembryonic antigen (CEA) levels, neoadjuvant chemotherapy, Clinical Risk Score (CRS) [23], type of liver surgery, transfusion data, overall duration of hospital stay, perioperative complications, radicality, site, and treatment of recurrence.

Overall survival and disease-free survival (DFS) were calculated from the date of liver resection. Complications or death occurring within 30 days or before discharge were considered perioperative. We defined a positive surgical margin as the presence of vital tumor along the line of transection.

After partial hepatic resection, patients routinely underwent a physical examination and determination of CEA level, abdominal/chest CT, or ultrasonography every
4 months for the first year, every 6 months the second year and once per year thereafter.

Statistical analyses were conducted using SPSS (version 15, SPSS Inc., Chicago, IL). Categorical variables are presented as number (percentage). Continuous variables are presented as median (range). Categorical variables were compared with the chi-square test; continuous variables were compared with the Mann-Whitney $U$ test. Actuarial survival was calculated using the Kaplan-Meier method from the date of resection of CLM, and differences in survival were examined using the log-rank test. $P<0.05$ (two-sided) was considered significant.

\section{Results}

Clinicopathological variables

Between January 2000 and June 2008, 308 patients underwent a partial hepatic resection for CLM; 201 patients met the study inclusion criteria, including 126 men $(63 \%)$ and 75 women $(37 \%)$. The median age was 65 (range, 30-86) years. The primary tumor was located in the colon in 114 patients (57\%) and rectum in 87 patients (43\%). After resection of the initial tumor, positive lymph nodes were present in 114 patients (57\%); synchronous liver metastases were identified in 78 patients $(39 \%)$. The median disease-free interval for the remaining 123 patients was 20 (range, 4-193) months from the time of resection of the colorectal tumor. The median CEA level was 16 (range, $1-1,292) \mathrm{ng} / \mathrm{ml}$ at the time of liver resection. In 16 patients $(8 \%)$, the CEA level exceeded $200 \mathrm{ng} / \mathrm{ml}$. The median number of metastases was one (range 1-8) with a median diameter of the largest metastases of 3 (range, 0.5-15) cm. The CRS was $\geq 3$ in 60 patients (30\%). Fifty-nine patients (31\%) were treated with neoadjuvant chemotherapy. AR was performed in 88 patients and NAR was performed in 113 patients. The clinicopathological features of the AR and NAR are compared in Table 1.

Surgical treatment

A single NAR was performed in 69 patients $(61 \%)$, whereas $44(39 \%)$ had two or more NAR simultaneously. A right hemihepatectomy was the most frequently performed AR (47 resections, 43\%) followed by left hemihepatectomy (15 resections, 14\%). Bisegmentectomies were performed in 18 patients (21\%; Table 2$)$.

\section{Outcome}

Table 3 presents the outcome of patients who underwent AR versus NAR. After AR, 32 patients (36\%) received a 
Table 1 Clinicopathological variables

\begin{tabular}{|c|c|c|c|}
\hline Variable & $\begin{array}{l}\text { Anatomical } \\
(n=88)\end{array}$ & $\begin{array}{l}\text { Nonanatomical } \\
(n=113)\end{array}$ & $P$ value \\
\hline Age (year) & $65(30-82)$ & $65(36-86)$ & 0.585 \\
\hline Gender (male) & $56(64)$ & $70(62)$ & 0.806 \\
\hline No. of tumors & $2(1-7)$ & $1(1-7)$ & 0.295 \\
\hline Size largest tumor $(\mathrm{cm})^{\mathrm{a}}$ & $4(1-15)$ & $3(1-7)$ & $<0.001$ \\
\hline Bilobar distribution & $20(23)$ & $32(28)$ & 0.369 \\
\hline $\mathrm{CEA}^{\mathrm{b}}$ & $16.4(1-1292)$ & $15.9(1-909)$ & 0.078 \\
\hline$>200 \mathrm{ng} / \mathrm{ml}$ & $10(12)$ & $6(5)$ & 0.113 \\
\hline \multicolumn{4}{|l|}{ Time to resection } \\
\hline Synchronous & $35(40)$ & $43(38)$ & 0.804 \\
\hline Metachronous & $53(60)$ & $70(62)$ & \\
\hline Disease-free interval (mo) & $24(4-93)$ & $17(4-193)$ & 0.430 \\
\hline \multicolumn{4}{|l|}{ Clinical risk score ${ }^{\mathrm{a}}$} \\
\hline $1-2$ & $57(66)$ & $82(73)$ & 0.241 \\
\hline $3-5$ & $30(34)$ & $30(27)$ & \\
\hline Neoadjuvant chemotherapy & $31(35)$ & $28(25)$ & 0.107 \\
\hline \multicolumn{4}{|l|}{ Site primary tumor } \\
\hline Colon & $55(63)$ & $59(52)$ & 0.144 \\
\hline Rectum & $33(37)$ & $54(48)$ & \\
\hline \multicolumn{4}{|l|}{ Tumor stage primary tumor } \\
\hline $0-2$ & $12(14)$ & $23(20)$ & 0.213 \\
\hline $3-4$ & $76(86)$ & $90(80)$ & \\
\hline \multicolumn{4}{|l|}{ Lymph node primary tumor } \\
\hline Positive & $45(51)$ & $69(61)$ & 0.159 \\
\hline Negative & $43(49)$ & $44(39)$ & \\
\hline
\end{tabular}

Missings: ${ }^{a}=2,{ }^{b}=4$

Data are numbers with percentages in parentheses or medians with ranges in parentheses unless otherwise indicated

blood transfusion. This was significantly lower after a NAR (23 patients, $20 \% ; P=0.012$ ). The transfused patients in the AR group received a median of 3 units of erythrocytes (range 1-6). In the NAR group, the median transfusion rate also was 3 units of erythrocytes (range 1-9), but with a larger range. The hospital stay was significantly shorter after NAR (7 (range, 1-26) days vs. 8 (range, 4-42) days; $P<0.001)$. There was no significant difference in mortality rate between the two groups. Insufficient capacity of the liver remnant was the cause of death in the two patients in the AR group. One patient in the NAR group died due to aspiration pneumonia. The median follow-up was 35 (range, 1-111) months in both groups. With respect to the median time to recurrence, the groups were comparable (AR group 9 (range, 1-46) months vs. 10 (range, 2-55) months in the NAR group; $P=0.802$ ). The DFS was similar for the AR and NAR groups: $56 \%, 38 \%, 30 \%$, and $60 \%, 39 \%, 32 \%$ at 1,3 , and 5 years, respectively $(P=0.441, P=0.81, P=0.599$; Fig. 1$)$. The pattern of
Table 2 Type of resection

\begin{tabular}{lcr}
\hline Liver resection & \multicolumn{2}{l}{ No. of resections } \\
\cline { 2 - 3 } & $(N=201)$ & $(\%)$ \\
\hline Nonanatomical $(n=113)$ & 69 & 61 \\
Single & 25 & 22 \\
Two & 13 & 12 \\
Three & 4 & 3 \\
Four & 2 & 2 \\
Five & & \\
Anatomical $(n=88)$ & 12 & 14 \\
S 2-3 & 6 & 7 \\
S 6-7 & 47 & 53 \\
Right hemihepatecomy & 15 & 17 \\
Left hemihepatectomy & 4 & 5 \\
Extended right hemihepatectomy & 1 & 1 \\
Extended left hemihepatectomy & 3 & 3 \\
Combination of anatomical resections ${ }^{\mathrm{a}}$ & 3 & \\
\hline$S$ segment & & \\
a seg 2-3 + seg 1 resection, seg 2-3 + seg $6-7$ resection &
\end{tabular}

Table 3 Outcome surgery

\begin{tabular}{lccr}
\hline Variable & $\begin{array}{l}\text { Anatomical } \\
(n=88)\end{array}$ & $\begin{array}{l}\text { Nonanatomical } \\
(n=113)\end{array}$ & $P$ value \\
\hline Blood transfusion & $32(36)$ & $23(20)$ & 0.012 \\
Hospital stay & $8(4-42)$ & $7(1-26)$ & $<0.001$ \\
Complications & $24(27)$ & $26(23)$ & 0.488 \\
In-hospital mortality & $2(2)$ & $1(1)$ & 0.421 \\
Positive resection margins & $8(9)$ & $12(11)$ & 0.728 \\
\hline
\end{tabular}

Data are numbers with percentages in parentheses or medians with ranges in parentheses unless otherwise indicated

recurrence did not differ between the two groups (Table 4). The 3-year intra hepatic recurrence rate was $37 \%$ in the AR group and $33 \%$ in the NAR group $(P=0.62)$. Seventeen patients in the AR group and 26 patients in the NAR group developed liver metastases limited to the liver. These patients received similar therapy (Table 4). The OS was $96 \%, 61 \%$, and $49 \%$ for the AR group and $97 \%, 65 \%$, and $39 \%$ for the NAR group at 1,3 , and 5 years, respectively $(P=0.715, P=0.611, P=0.989$; Fig. 2$)$.

\section{Discussion}

This study demonstrated no significant difference in outcome between patients with CLM after anatomical or NAR. The 5-year disease-free (AR 30\% vs. NAR 32\%) and OSs (AR 49\% vs. NAR 39\%) in our study is consistent with the literature [2, 24-28]. 


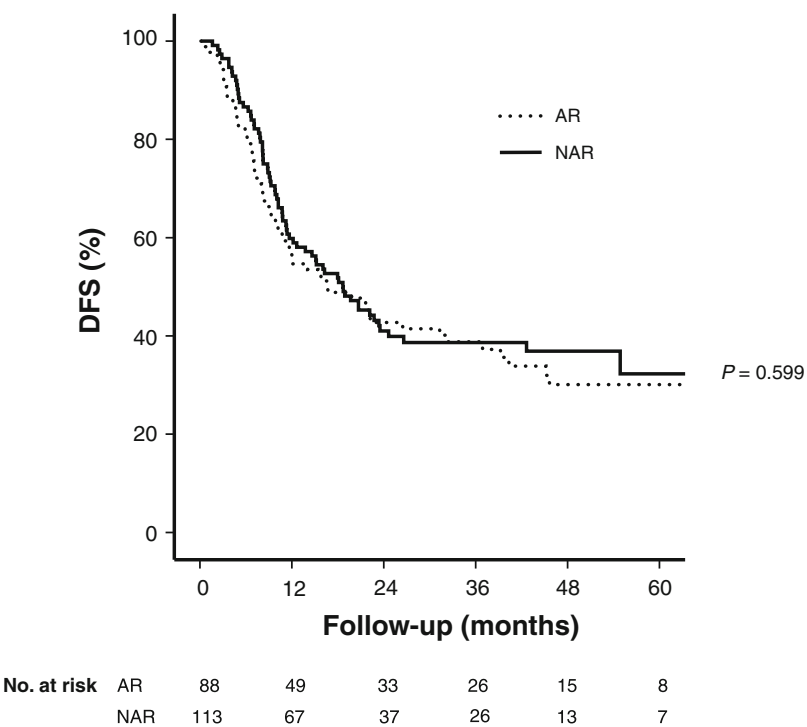

Fig. 1 Disease-free survival stratified by surgical procedure. Median DFS was 16.7 months in the AR group and 18.7 months in the NAR group. The 5-year DFS rate was 30 and $32 \%$, respectively $(P=0.599)$

Table 4 Patterns of recurrence and treatment modality

\begin{tabular}{lccc}
\hline & $\begin{array}{l}\text { Anatomical } \\
(n=88)\end{array}$ & $\begin{array}{l}\text { Nonanatomical } \\
(n=113)\end{array}$ & $P$ value \\
\hline Location recurrence & & & 0.156 \\
Liver & $17(30)$ & $26(38)$ & \\
Liver + lung & $10(18)$ & $4(6)$ & \\
Liver + elsewhere & $2(2)$ & $5(7)$ & \\
Elsewhere & $28(49)$ & $34(49)$ & \\
Therapy liver metastases & & & \\
No therapy & $1(6)$ & $2(8)$ & \\
Systemic therapy & $9(53)$ & $8(32)$ & \\
Local therapy & $7(41)$ & $15(60)$ & \\
Resection & 3 & 10 & \\
RFA & 2 & 3 & \\
STBR & 1 & 2 & \\
Liver perfusion & 1 & 0 & \\
\hline
\end{tabular}

$R F A$ radiofrequency ablation; $S T B R$ stereotactic body radiation Data are numbers with percentages in parentheses or unless otherwise indicated

The major drawback is the retrospective nature of this study. Randomization would be difficult in this patient group, because the technique for liver resection is a tailormade approach based on the size, number, location, and distribution of the metastases. In addition, the consideration between conservation of liver parenchyma, complete surgical tumor clearance, and complications is of importance in this decision. Although patients were not randomized, the basic characteristics were similar as shown in Table 1.

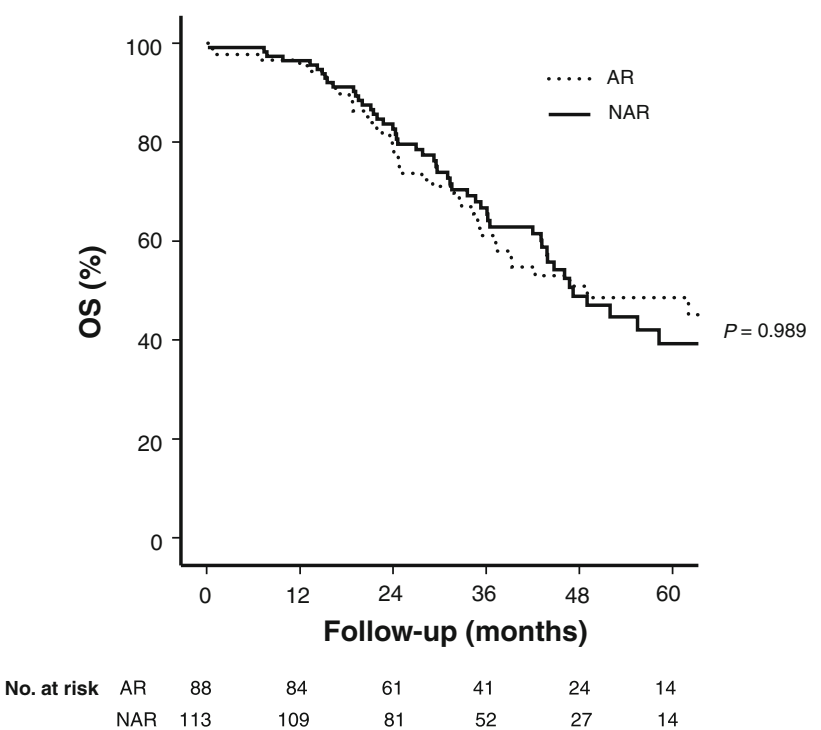

Fig. 2 Overall survival stratified by surgical procedure. Median OS was 49 months in the AR group and 47.2 months in the NAR group. The 5-year OS rate was 49 and $39 \%$, respectively $(P=0.989)$

Liver parenchymal-sparing surgery is already frequently used for CLM for several reasons. Functional hepatic reserve must be considered for any liver resection; its significance increases in the context of neoadjuvant chemotherapy, which is used to downsize the tumor load, making more patients eligible for surgery [29]. However, although chemotherapy increases resectability, it is associated with significant hepatic changes, such as hepatic sinusoidal obstruction, periportal inflammation, and steatohepatitis, which can affect patient outcome [15]. Specifically, chemotherapy-associated steatohepatitis is associated with the risk of progressive hepatic failure and death after resection [16]. Therefore, maximizing the amount of residual liver parenchyma is of considerable importance in patients who have had chemotherapy.

Moreover, surgical stress can be reduced by nonanatomical resections, which may affect perioperative morbidity and mortality $[14,25]$. Several studies reported significant shorter operating times and significant less blood loss after NAR [25, 26, 28]. This also is seen in our study population. Patients who underwent AR received significant more blood transfusions than the patients after NAR (AR $36 \%$ vs. NAR $20 \% ; P=0.012$ ). In our series, there were three deaths within 30 days of surgery: two in the AR group, and one in the NAR, which was not significantly different. There are studies suggesting more postoperative deaths in the AR group [2, 25, 26, 28]. It is important to note that postoperative mortality is a rare event and that these studies are not powered to compare this.

The possibility to treat recurrent CLM with local therapy, such as repeated hepatectomy [17], RFA [11], or STBR [30] is a great benefit of the parenchymal sparing 
method. In our study, disease recurrence in the liver was similar for both AR and NAR (51\%). The reintervention rate for CLM was higher in the NAR group (AR $41 \%$ vs. NAR $60 \%$ ). Although this number does not reach significance, probably due to the small numbers, our findings suggest that local treatment for intrahepatic recurrences is more often possible in the parenchymal-sparing method. Our findings are consisted with the literature, which states that reinterventions for CLM increases the survival after disease recurrence [31-33]. For this reason, close surveillance of patients after NAR is essential. One of the possible disadvantages of NAR reported in the literature by DeMatteo et al. [24] is the higher incidence of positive resection margins. In more recently published literature, it is advocated that a resection margin $<1 \mathrm{~cm}$ is no longer a contraindication for curative resection. Moreover recent literature suggests that size of surgical margin does not correlate significantly with DFS or OS; even the need for $\mathrm{R} 0$ resections is being discussed [34, 35]. In a study by de Haas et al., the 5-year OS was similar for patients after a $\mathrm{R} 0$ or a R1 resection (61 vs. $57 \% ; P=0.27$ ), although the recurrence was higher in the R1 group (28 vs. 17\%; $P=0.004$ ) [6]. In our study, the R1 resection rate was $9 \%$ in the AR group and $11 \%$ in the NAR group, which is comparable to the literature $[6,27]$. The concept of performing limited NAR with narrow margins is supported by the fact that micrometastases in the liver parenchyma surrounding CLM are rare and are primarily confined to the immediate surrounding area of the tumor border [36, 37].

The second possible drawback of NAR, which is postulated in the literature ([24]), is the lack of vascular control. This is the opposite of what was published during the past years. Blood loss and blood transfusions are reported to be significantly less during and after NAR, which is confirmed by our results $[25,26,28]$.

In contrast to CLM, some studies report AR to be superior to NAR in HCCs [18-20]. This difference may be explained by the variation in disease biology seen in primary versus metastatic liver tumors. Metastatic liver lesions develop from blood-borne tumor cells circulating throughout the body. AR may not offer the same advantage for these lesions as for HCC, which arise within a segment of the liver and might benefit from the removal of the complete functional liver unit.

Multiple studies have been conducted to investigate which resection is favorable for patients with CLM: anatomical or nonanatomical. Most authors similarly conclude that there is no significant difference between AR and NAR in disease-free and OSs. A disadvantage of the majority of studies is that the patient characteristics are not comparable between the two groups regarding tumor size and number, nodal status of the primary tumor, disease-free interval, and CEA blood levels $[2,14,25,26]$. Our study contributes to this discussion due to the use of the CRS in which the previous described characteristics are incorporated. The CRS is the same for the AR and NAR, which indicates that the groups are comparable.

Furthermore, the use of different neoadjuvant chemotherapy regimens during the years makes it difficult to compare the results of the studies [2, 14, 26-28]. We started our patient selection after 2000, because Irinotecan and Oxaliplatin were added to the chemotherapeutic arsenal from this year forward, and all patients were treated with effective chemotherapeutics.

We conclude that with a comparable complication rate, less blood transfusions, a significantly shorter hospital, and comparable disease-free and OS rates, a NAR is a safe technique for the resection of CLM.

Open Access This article is distributed under the terms of the Creative Commons Attribution Noncommercial License which permits any noncommercial use, distribution, and reproduction in any medium, provided the original author(s) and source are credited.

\section{References}

1. Stangl R et al (1994) Factors influencing the natural history of colorectal liver metastases. Lancet 343(8910):1405-1410

2. Zorzi D et al (2006) Comparison between hepatic wedge resection and anatomic resection for colorectal liver metastases. J Gastrointest Surg 10(1):86-94

3. Simmonds PC et al (2006) Surgical resection of hepatic metastases from colorectal cancer: a systematic review of published studies. Br J Cancer 94(7):982-999

4. Geoghegan JG, Scheele J (1999) Treatment of colorectal liver metastases. Br J Surg 86(2):158-169

5. Muratore A et al (2009) Resection margin and recurrence-free survival after liver resection of colorectal metastases. Ann Surg Oncol 17(5):1324-1329

6. de Haas RJ et al (2008) R1 resection by necessity for colorectal liver metastases: is it still a contraindication to surgery? Ann Surg 248(4):626-637

7. Saltz LB et al (2000) Irinotecan plus fluorouracil and leucovorin for metastatic colorectal cancer. Irinotecan Study Group. N Engl J Med 343(13):905-914

8. Adam R et al (2004) Rescue surgery for unresectable colorectal liver metastases downstaged by chemotherapy: a model to predict long-term survival. Ann Surg 240(4):644-657 discussion 657-658

9. Azoulay D et al (2000) Resection of nonresectable liver metastases from colorectal cancer after percutaneous portal vein embolization. Ann Surg 231(4):480-486

10. Hemming AW et al (2003) Preoperative portal vein embolization for extended hepatectomy. Ann Surg 237(5):686-691 discussion 691-693

11. de Meijer VE et al (2006) Radiofrequency ablation in patients with primary and secondary hepatic malignancies. J Gastrointest Surg 10(7):960-973

12. Wong SL et al (2010) American Society of Clinical Oncology 2009 clinical evidence review on radiofrequency ablation of hepatic metastases from colorectal cancer. J Clin Oncol 28(3): 493-508

13. Mendez Romero A et al (2006) Stereotactic body radiation therapy for primary and metastatic liver tumors: a single institution phase I-II study. Acta Oncol 45(7):831-837 
14. Gold JS et al (2008) Increased use of parenchymal-sparing surgery for bilateral liver metastases from colorectal cancer is associated with improved mortality without change in oncologic outcome: trends in treatment over time in 440 patients. Ann Surg 247(1):109-117

15. Vauthey JN et al (2006) Chemotherapy regimen predicts steatohepatitis and an increase in 90-day mortality after surgery for hepatic colorectal metastases. J Clin Oncol 24(13):2065-2072

16. Zorzi D et al (2007) Chemotherapy-associated hepatotoxicity and surgery for colorectal liver metastases. Br J Surg 94(3):274-286

17. van der Pool AE et al (2009) Local treatment for recurrent colorectal hepatic metastases after partial hepatectomy. J Gastrointest Surg 13(5):890-895

18. Ueno $\mathrm{S}$ et al (2008) Efficacy of anatomic resection vs nonanatomic resection for small nodular hepatocellular carcinoma based on gross classification. J Hepatobiliary Pancreat Surg 15(5):493-500

19. Wakai $\mathrm{T}$ et al (2007) Anatomic resection independently improves long-term survival in patients with T1-T2 hepatocellular carcinoma. Ann Surg Oncol 14(4):1356-1365

20. Hasegawa $\mathrm{K}$ et al (2005) Prognostic impact of anatomic resection for hepatocellular carcinoma. Ann Surg 242(2):252-259

21. Couinaud C (1957) Etudes anatomiques et chirgicales. Masson \& Cie, Paris

22. Strasberg SM (2005) Nomenclature of hepatic anatomy and resections: a review of the Brisbane 2000 system. J Hepatobiliary Pancreat Surg 12(5):351-355

23. Fong Y et al (1999) Clinical score for predicting recurrence after hepatic resection for metastatic colorectal cancer: analysis of 1001 consecutive cases. Ann Surg 230(3):309-318 discussion 318-321

24. DeMatteo RP et al (2000) Anatomic segmental hepatic resection is superior to wedge resection as an oncologic operation for colorectal liver metastases. J Gastrointest Surg 4(2):178-184

25. Kokudo $\mathrm{N}$ et al (2001) Anatomical major resection versus nonanatomical limited resection for liver metastases from colorectal carcinoma. Am J Surg 181(2):153-159
26. Stewart GD et al (2004) The extent of resection influences outcome following hepatectomy for colorectal liver metastases. Eur J Surg Oncol 30(4):370-376

27. Finch RJ et al (2007) Effect of type of resection on outcome of hepatic resection for colorectal metastases. Br J Surg 94(10): $1242-1248$

28. Sarpel U et al (2009) Does anatomic versus nonanatomic resection affect recurrence and survival in patients undergoing surgery for colorectal liver metastasis? Ann Surg Oncol 16(2):379-384

29. Adam R et al (2009) Patients with initially unresectable colorectal liver metastases: is there a possibility of cure? J Clin Oncol 27(11):1829-1835

30. van der Pool AE et al (2010) Stereotactic body radiation therapy for colorectal liver metastases. Br J Surg 97(3):377-382

31. Yamamoto $J$ et al (1999) Repeat liver resection for recurrent colorectal liver metastases. Am J Surg 178(4):275-281

32. Shaw IM et al (2006) Repeat hepatic resection for recurrent colorectal liver metastases is associated with favourable longterm survival. Br J Surg 93(4):457-464

33. Petrowsky $\mathrm{H}$ et al (2002) Second liver resections are safe and effective treatment for recurrent hepatic metastases from colorectal cancer: a bi-institutional analysis. Ann Surg 235(6):863-871

34. Bodingbauer $M$ et al (2007) Size of surgical margin does not influence recurrence rates after curative liver resection for colorectal cancer liver metastases. Br J Surg 94(9):1133-1138

35. Pawlik TM et al (2005) Effect of surgical margin status on survival and site of recurrence after hepatic resection for colorectal metastases. Ann Surg 241(5):715-722 discussion 722-724

36. Yamamoto $\mathrm{J}$ et al (1995) Pathologic support for limited hepatectomy in the treatment of liver metastases from colorectal cancer. Ann Surg 221(1):74-78

37. Kokudo $\mathrm{N}$ et al (2002) Genetic and histological assessment of surgical margins in resected liver metastases from colorectal carcinoma: minimum surgical margins for successful resection. Arch Surg 137(7):833-840 